\title{
3D Body Scanning - An Important Tool for Digital Archiving of Cosmetic Surgery Procedures
}

\author{
David B. STEFAN ${ }^{1}$, David A. GILBERT ${ }^{2}$ \\ ${ }^{1}$ Novaptus Systems Inc., Chesapeake VA, USA; \\ 2 The Hague Plastic and Cosmetic Surgery Center, Norfolk VA, USA \\ DOI: $10.15221 / 17.139$ http://dx.doi.org/10.15221/17.139
}

\begin{abstract}
Background

At the end of 2002, a 3D whole-body white light scanner was introduced into a cosmetic surgery practice in Norfolk, Virginia. The stated purpose was to investigate this device as an aid for evaluating body contouring procedures. For the first time, the surgeon could pre-operatively scan a patient, measure "body areas of interest," and examine in detail the patient's 3D body contours to help plan for the pending surgical procedure. Multiple, custom designed measurement templates were then applied to the 3D body model to extract measurements pertinent to the surgical procedure. The pre-operative 3D body model was stored. Post-operative scans of the patient would then be taken periodically, and measured with the same measurement templates. The difference in measurements, including volume and surface area document the physical changes of patient's body resulting from the procedure. Many cosmetic surgery patients return for additional procedures over the course of years. The patient's 3D digital archive provides the surgeon with the ability to consult previous 3D body models and evaluate the measurement changes of past procedures. These unique insights, combined with a current 3D scan and traditional medical information form a contiguous and robust foundation for assessing the pending surgical procedure.
\end{abstract}

\section{Methods}

Three case examples are presented. Case 1 considers multiple cosmetic procedures performed in a close succession and the 3D scans and measurement templates used for pre-operative and postoperative evaluation. Case 2 presents an example 3D body scans of sequential cosmetic surgical procedures to replace and rebuild the left and right breasts of a subject. Case 3 involves multiple cosmetic procedures performed on the same patient over a period of years. In each case, the patient's 3D digital archive played an important role in documenting each surgical procedure and provided a platform to evaluate the contours of the patient for the pending surgery.

\section{Discussion}

A 3D whole-body scan is an independent entity. It represents the accurate physical appearance of the subject standing in the scan chamber as of the date and time of scan acquisition. A cosmetic surgery patient may undergo multiple procedures at once, such as breast reduction and abdominoplasty. The result of each procedure can be measured by applying separate customized measurement templates to the post-operative 3D body model. The patient may also undergo a sequence of procedures over time. Each post-operative 3D body model can be evaluated independently. The entire set of preoperative and post-operative 3D body scans form a historical 3D digital archive and can be appended to the traditional patient medical record.

\section{Conclusion}

The utility of a 3D whole-body scanner is in its ability to create an accurate 3D body model of the subject within the scan chamber, thereby creating a permanent 3D record once stored. A "clean" 3D scan documents the subject's physical body. Measurements can be extracted at any time as appropriate, but the archived 3D body model is the important record. Subsequent body scans can be measured and compared to the initial body scan. Many cosmetic surgery procedures result in immediate physical changes to the body. Each post-operative scan documents these changes. Successive post-operative scans form the patient's 3D digital archive. This archive can serve as the basis for further surgical planning, validating surgical results, or can be used as a tool for independently assessing surgical outcomes. 


\section{Introduction}

The reader is referred to the references for a complete background and history of the 3D whole-body booth scanners that have been deployed at The Hague Center for Cosmetic Surgery, in Norfolk, Virginia, USA. The following paragraphs offer only a highly condensed version.

IRB NUMBER 05-09-FB-0235 Eastern Virginia Medical School provides the basis for this research and presentation, to which the subjects have signed the appropriate consent forms.

A 3D whole-body booth-type scanner, in its original mechanical sensor (the combination of projector and camera) configuration, and its solid-state sensor successors has been employed at The Hague Center for Cosmetic Surgery in Norfolk, Virginia since November of 2002.

During the span of these years, many patients have been scanned pre-operatively and post-operatively, often on a periodic basis as part of routine follow-up visits. The series of pre-operative and postoperative scans form the patient's 3D digital archive.

The original purpose of utilizing a 3D whole-body scanner was to investigate whether the 3D body models acquired by the scanner, along with the scanner measurement software could act as an aid to the surgeon for evaluating preoperative body contours.

Measurements could be selected from the vast array offered by the measurement software, including mathematical combinations of measurements constructed using "Reverse Polish Notation." The selections could be programmed into a "measurement extraction template." This would be applied to the 3D body model created by scanning the individual within the scan chamber.

This programmable measurement capability meant that, with experiment, measurement profiles could be constructed which could extract valuable contour information for specific cosmetic surgery procedures. The same measurement template could then be applied to the post-operative body model, documenting the physical changes and validating the surgical procedure.

It didn't occur to us until later that the important element wasn't the extraction of specific measurements from the 3D body model, useful as they were, but the 3D body model itself. Once a usable 3D body model has been created, it can be archived for later retrieval and use. The 3D body model can also be exported using multiple, standard 3D data formats for use in many commonly available CAD modeling programs. This allows the ability to manipulate the 3D body model and simulate or predict the outcome of various cosmetic surgical procedures such as breast augmentation and breast reduction.

Multiple measurement profiles can be applied to the 3D body model. In effect, the 3D body model is always "reusable." One can apply an existing measurement template, evaluate the returned measurements, then add or eliminate measurements. This can continue until the profile extracts pertinent measurement information from the desired area of the patient's body, as represented by the patient's 3D body model. The 3D body model houses all the linear, contoured, circumferential measurements and heights of those measurements, as well as surface area and volume information. The programmable measurement extraction profile simply "unlocks" the desired measurements from the 3D body model. The 3D body model always remains unchanged.

If new measurements are considered, they can be introduced into an existing measurement template, or a new template can be created. 3D body models from the distant past can be retrieved, and the new measurements can be extracted, tabulated and compared.

We have utilized several generations of scanning technology since the first, very expensive and bulky 16-foot long by 8-foot wide by 7-foot high 3D body scanner was introduced into the practice in November of 2002. Yet the 3D body models acquired from the original scanner can still be retrieved and measured with the latest version of the scanner measurement software. This implies continuity, as patients that were scanned using the first 3D scanning system could continue to be scanned with later scanning systems.

\section{The leap to 3D from 2D photographic archives and its advantages}

The reason that this is consequential is that it represents a change in the traditional method that the cosmetic surgeon uses to keep patient records. In 2002, when the first 3D body scanner was employed, the common method for documenting the patient's pre-operative body was to take analog photographs using $35 \mathrm{~mm}$ film. 
The photographs were developed as prints and slides. These were carefully cataloged. When the surgeon wished to give a presentation, he often used a slide carousel that projected the image on the slide onto a presentation screen. Of course, slides and photographs age over time and are not ideal methods for preserving images of surgical work.

In the mid-2000's, digital cameras became available. These began to supplant analog photography. Photographs could be processed by computer, stored in digital format, and printed on photographic paper. Digital storage allowed preservation of the images. The yellowing of photographs that occurs over time, and dulling of images on photographic "negatives" became a thing of the past.

However, "before" and "after" pictures taken by a digital camera lacked consistent perspective. They could be taken at different angles and at different distances. They could also be taken with the patient being in different postures. The surgeon wasn't concerned about this, though. The intent of the digital photographs was to emphasize physical changes to the body resulting from the surgical procedure.

The photographic images, whether displayed on a computer monitor or presented in print, were still two-dimensional. Because they were taken from unknown distances, even linear measurements were impossible to produce.

The original body scanner took a meaningful step in the transition away from two-dimensional digital archiving by introducing a three-dimensional storage format. Theoretically, the individual stands in a fixed position within the scan chamber. The feet are separated from the torso by means of standing over footprints affixed to the floor of the 3D scanner. The arms are lifted away from the torso by the individual grasping handles located within the sides of the scan chamber.

Assuming the 3D scanner is in proper calibration, the result of acquiring a 3D scan is an accurate 3D body model faithfully representing the surface contours of the individual within the scan chamber. Moreover, the 3D body model is created using a fixed perspective when presented on the scanner computer screen. Scanner software settings allow the 3D body model and the images captured by the sensor cameras to be automatically saved to storage hardware.

Once the 3D body model has been produced, measurement templates can be applied. These measurements are extracted from the 3D body model, displayed on the computer monitor and can be exported as a file in any number of formats for further processing, including formats compatible with popular spreadsheet programs.

The image of the 3D body model on the computer monitor can be rotated and panned (moved up, down, left and right without affecting viewing distance). The image can also be magnified or reduced. Together, these capabilities allow the 3D body model to be viewed at all angles.

Because the 3D body model is created from a fixed perspective, that is, from a fixed position within the scan chamber, subsequent scanning of the same individual results in 3D body models that can be measured in the same fashion as the pre-operative 3D body model. The measurement differences between 3D body models can be tabulated and stored. 3D viewing and measurement extraction transcends simple visual comparison between traditional 2D photographs.

\section{Early 3D scanning technology, its deficiencies and constraints}

The sensor technology utilized in the 3D body scanners up to 2013 employed white light in a structured pattern that was projected onto the individual within the scan chamber. This pattern was generated by horizontal grid patterns that were placed before the light source of each sensor head (the earliest scanner had 4 moving sensors, later scanners used 12 and 16 sensors in fixed locations). The projected light would be reflected into the sensor cameras, and images at the appropriate angles and height would be combined to construct the 3D body model. These 3D scanner versions did not offer the capability to view the 3D body model in color (the cameras used in the 12 and 16 sensor 3D scanner versions had color capability but it introduced distortion errors in the construction of the 3D body model, so the color feature was disabled).

The use of white light as a projection technology required certain constraints. As white light is absorbed by black material, a considerable effort was necessary to ensure no outside light entered the scan chamber during the scan acquisition. This included darkening the room that housed the 3D scanning system. Moreover, dark undergarments or garments that contained combinations of light and dark could not be worn during the scan process. 
It is important to note that these 3D body scanners create a 3D surface image only, so care is required in to prepare the individual for scan acquisition. These preparations are not onerous, but need to be followed for the creation of a 3D body model that can be faithfully measured.

The individual must be scanned either in light-colored, (white, beige, gray) closely fitting garments, or in the nude. The hair must be held above the neckline. Jewelry that could scatter the projected light must not be worn. These are simple guidelines. If an individual's hair was down below the neckline during scan acquisition, if would be visible on the 3D body model. A neck measurement extraction would yield the circumference value around the hair. If a male was scanned in boxer shorts, the thigh circumferences would yield a value that spanned the circumference of the boxer shorts, and not the true thigh circumference.

\section{D body scanning in a clinical environment}

In the cosmetic practice, a separate room with a windowless door was used to house the scanner. The patients would be instructed as to how the scanner worked. The trained operator (nurse or administrator) would leave the room after asking the patients to disrobe, enter the scan chamber and close the entrance curtain. Less than five minutes later, the operator would knock on the door, and ask if the patient was in the chamber and ready to be scanned. If the answer was affirmative, the operator would check that the curtain was drawn tight, making certain no outside light would enter the scan chamber and ask the patient if they are standing in the proper position.

The operator then initiates the scan program, and the patient within the scan chamber presses a button with their thumb on the right arm handle to begin the scanning process. The scan acquisition process completes within 30 seconds. It requires another 60 seconds to reduce the raw images into a 3D body model. This 3D body model is then displayed on the computer monitor. At this point, any obvious errors could be detected by the operator (incorrect posture, movement during scan acquisition, etc.). If present, the operator identifies and addresses these issues by asking the patient to be re-scanned. In general, if the simple guidelines are followed, the initial scan is usually acceptable.

\section{Three body model viewing modes}

In late 2013, sensors were introduced that employed near-field infrared technology utilized in motionsensing game consoles (Xbox, Wii, etc.). This improved the scan acquisition process. A darkened scan chamber was no longer a requirement, though the enclosed booth was kept for privacy purposes. Guidelines for hair, separation of the arms and legs from the torso, and close-fitting clothes (if any) were still required to ensure creation of an accurate 3D body model.

The near-field infrared 3D body scanners offered "true color."

There are three modes available for display of the 3D body model: "Point-Cloud," "Surface," and "Color." Each viewing mode has its purpose. The "point-cloud" mode depicts the thousands of 3D points that collectively comprise the surface outline of the 3D body model. This viewing mode offers little surface detail, but is useful when viewing circumferential measurements. It can be considered a viewing mode that offers "see-thru" capability.

"Surface" mode connects the points that comprised the surface of the 3D body model together. In effect, it creates a marble-like statue. This allows the viewing of detailed surface contours.

"Color" mode presents the 3D body model in the view of the underlying sensor camera technology. When 3D scanners using white light for scan acquisition are utilized, "color" mode displays the 3D body model in the black and white images used for its creation. These images often contain a structured light pattern. Hence the 3D body model, depending on the scanner version, appears to have horizontal stripes of varying intensity. 3D body models created by near-field infrared sensors are shown in color. This mode can be utilized for viewing pre-operative markings on the patient's surface pending the surgical procedure.

Figure 1 presents the three modes for viewing the 3D body model. 


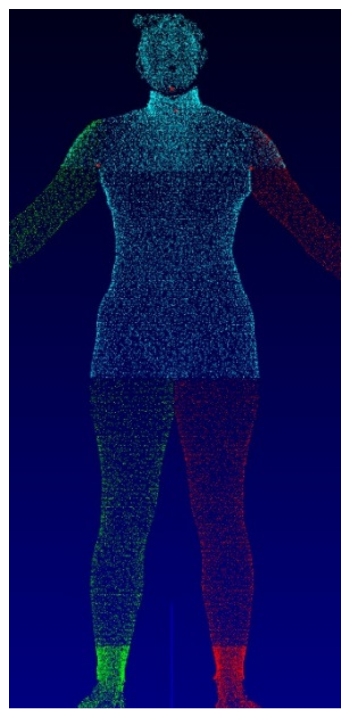

"Point-Cloud" Mode

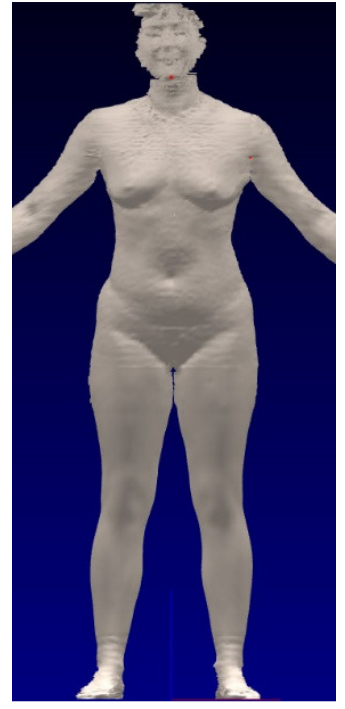

"Surface Mode"

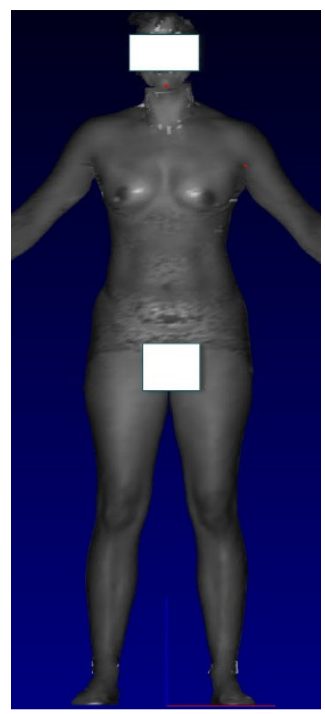

“Color” Mode

Figure 1. The Three Viewing Modes for Display of the 3D Body Model

As camera sensor technology continues to evolve, finer color resolution will be commonplace, but it will not supplant other viewing modes.

The important point is the creation of an accurate 3D body model, regardless of the underlying sensor technology. 3D body models that were created by the earliest 3D body scanner can still be successfully measured by the latest scanner measurement software.

\section{Case examples}

Case 1 presents a patient that underwent two cosmetic surgery procedures in close succession. The first procedure was a breast reduction. An abdominoplasty followed immediately afterwards. Thus, there are two procedures affecting different areas of the body. Two separate measurement templates were used for preoperative evaluation, one to extract measurements related to the breasts and upper body, the other to extract measurements from the lower portions, starting at the waist.

Figure 2 displays a breast measurement template applied to the pre-operative 3D body model. This image is in surface mode. The yellow lines represent the linear, contoured linear and circumferential measurements extracted by the measurement profile. These measurements are tabulated and presented in the same fashion as they are displayed on the computer monitor.

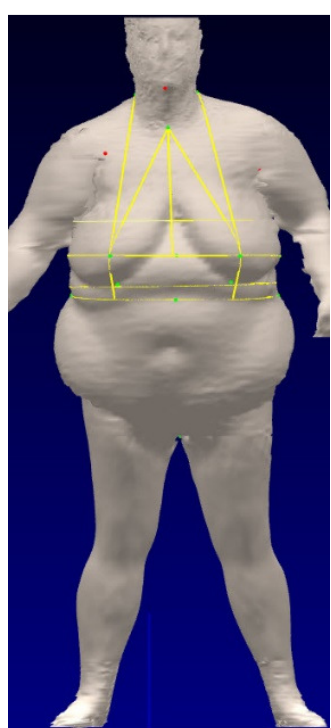

Pre-operative 3D Body Model in "Surface Mode"

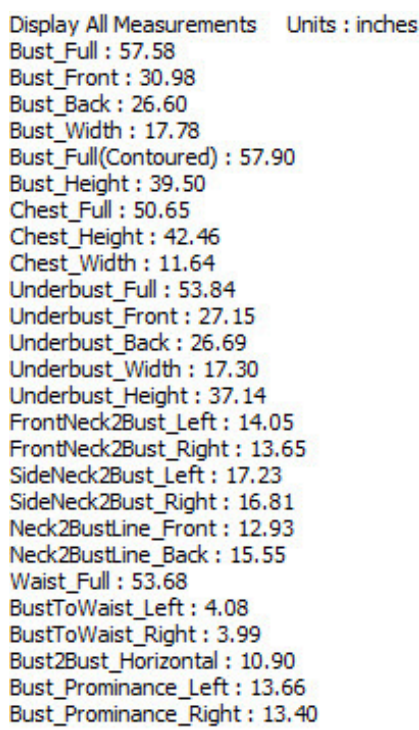

Breast Region Measurements Extracted

Figure 2. Pre-operative Patient 3D Body Model in Surface Mode and Extracted Measurements 
The measurement profile applied to the pre-operative 3D body model has been used for evaluation of surgical cases involving the breasts (augmentation, reduction, breast-lift, reconstruction). The measurements in this template help identify breast asymmetries prior to surgery.

Figure 3 presents a lower body measurement template applied to the same preoperative 3D body model. The 3D body model is depicted in point-cloud mode. Note that one can observe the circumferences of some of the extracted measurements, particularly around the legs (thigh, knee and calf). The surface and color modes would not allow a transparent view of these circumferences.

It is true that these measurement templates could be combined into one large profile, but the measurements would need to be separated to address the specific changes caused by the breast reduction surgery and the abdominoplasty. By applying two separate measurement extraction profiles to the same 3D body model, measurement changes are specific to the procedure.

The 3D body model is not affected by application of a measurement template.

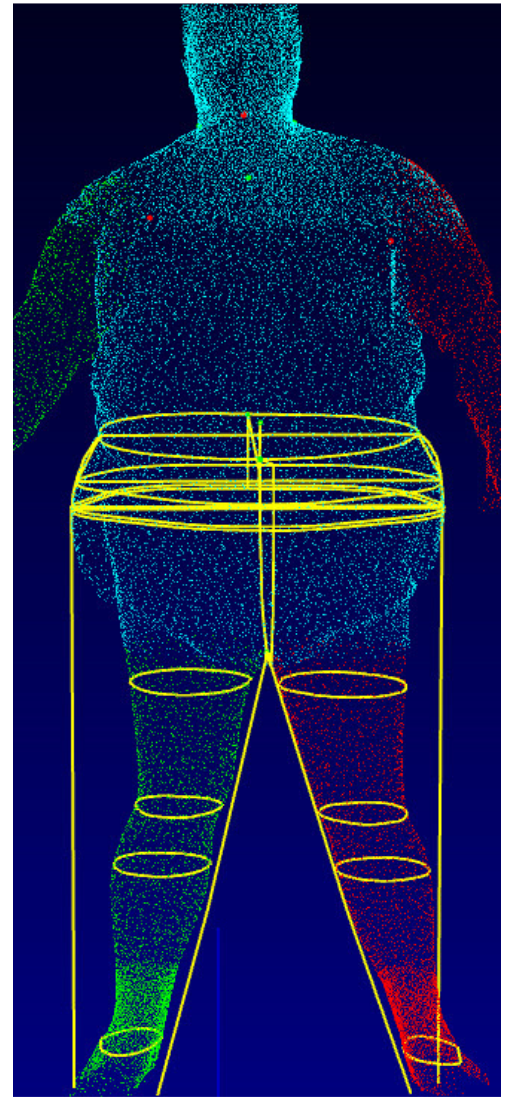

Pre-operative 3D Body Model "Point-Cloud"

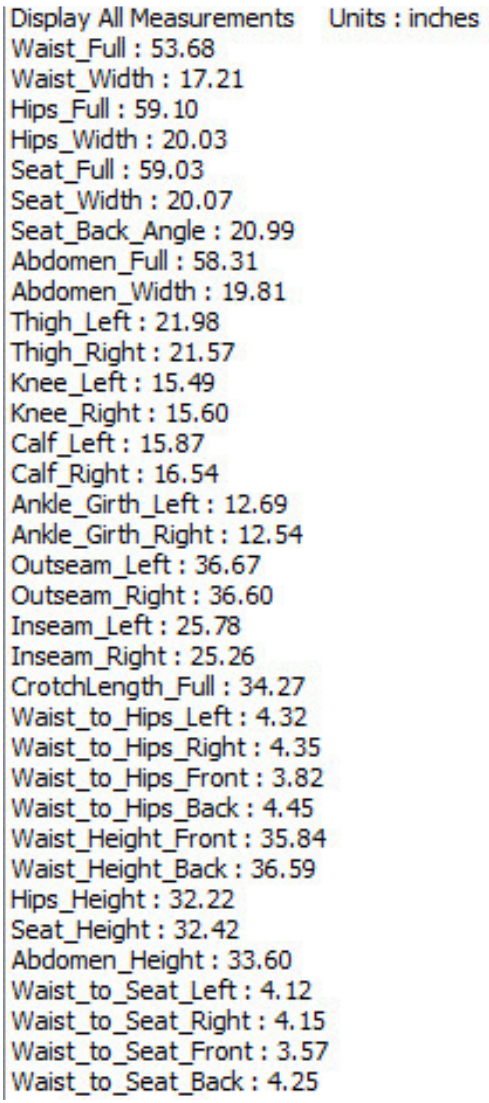

Lower-body Measurements Extracted

Figure 3. Pre-operative Patient 3D Body Model in "Point-Cloud" Mode and Extracted Measurements

Note that the measurements displayed for both extractions are in units of inches. The measurement unit is selectable between inches, centimeters and millimeters.

The lower-body measurement template has been used for evaluation of abdominoplasty, circumferential pannulectomy, thigh-lifts and gluteal and calf implant surgical procedures.

Many of the measurements offered by the scanner measurement software find their origins in the clothing industry. Measurements names such as "bust," "outseam," and "inseam," are familiar in such a setting. We have found several clothing measurements to be of interest in the medical world. One such measurement is "Crotchlength_Full," another is "Seat_Back_Angle."

In effect, these are body contouring measurements that reflect important changes to the physical shape of the body that occur as a result of a lower-body surgical procedure. 
Figure 4 displays the post-operative 3D body model acquired 3 months post-surgery. The image on the left depicts the application of the breast measurement template. The image on the right is the application of the lower body measurement template. Both are surface images.

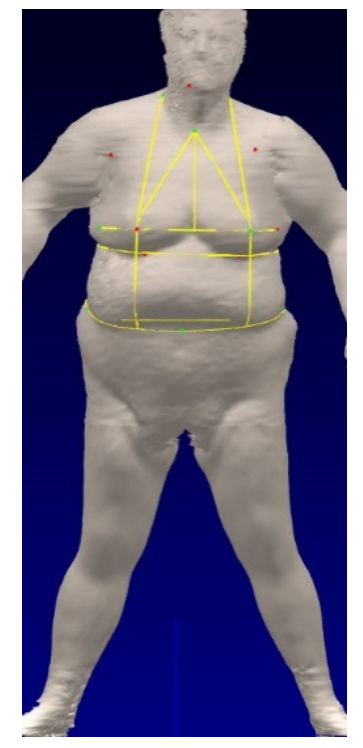

3-Mo Post-Operative 3D Body Model with Bust Measurement Template

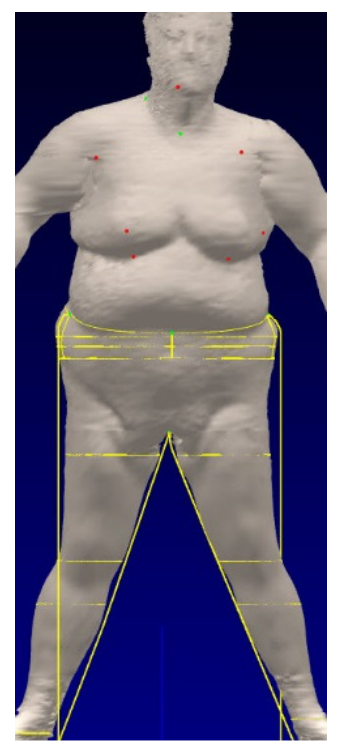

3-Mo Post-Operative 3D Body Model with Lowerbody Measurement Template

Figure 4.3 Month Post-Operative Body Model with Separate Measurement Templates Applied

Figure 5 tabulates the measurements extracted from the pre-operative and post-operative 3D models for the breast measurement template.

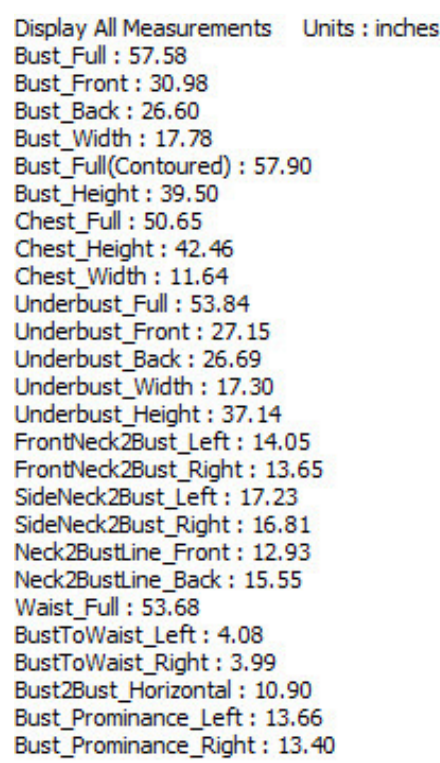

Pre-Operative Breast Area Measurements

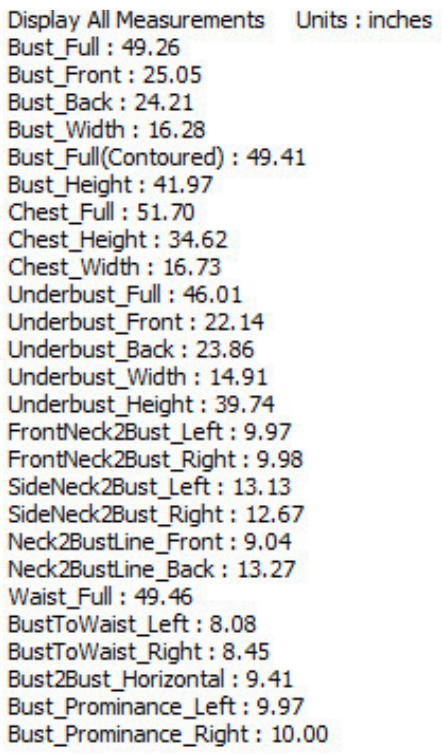

3-Mo Post-Operative Measurements

Figure 5. Pre- and Post-Operative Breast Area Measurement Comparisons

Here we see the advantage of applying two separate measurement templates to the preoperative and post-operative 3D body models. By quick observation of the measurement differences extracted by the breast area measurement template, one can note that the bust circumference has been reduced by over 8 inches. Measurements related to breast symmetry, such as "FrontNeck2Bust" and "Bust_Prominance" left and right indicate that they are nearly equal. 
Figure 6 presents the lower body measurements extracted from the pre-operative and post-operative $3 \mathrm{D}$ body models.

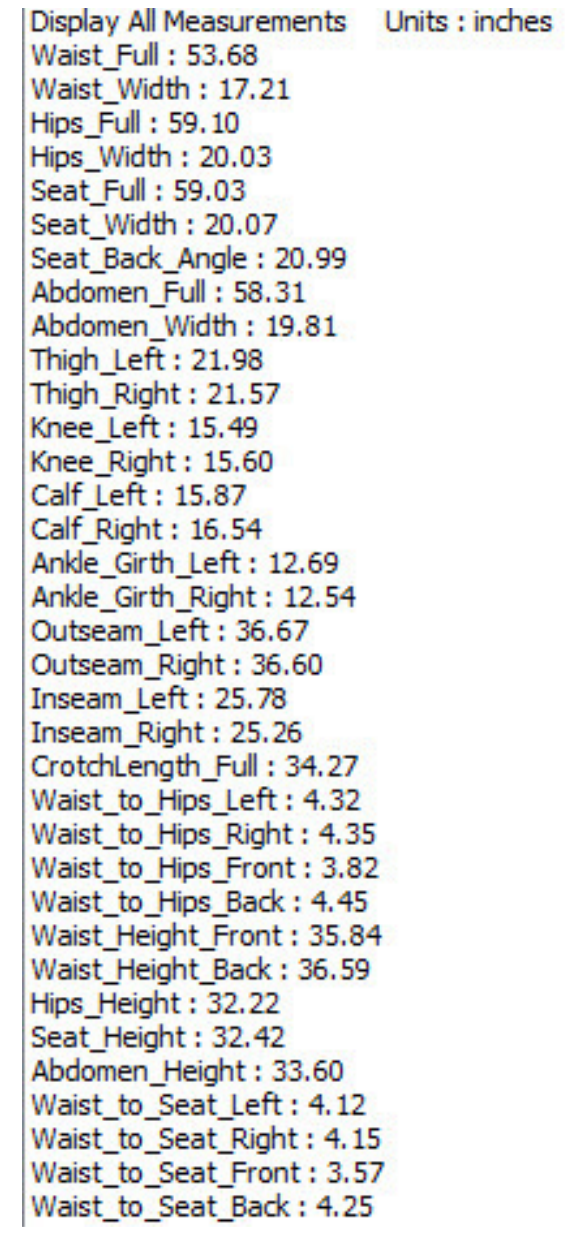

Pre-Operative Lower Body Measurements

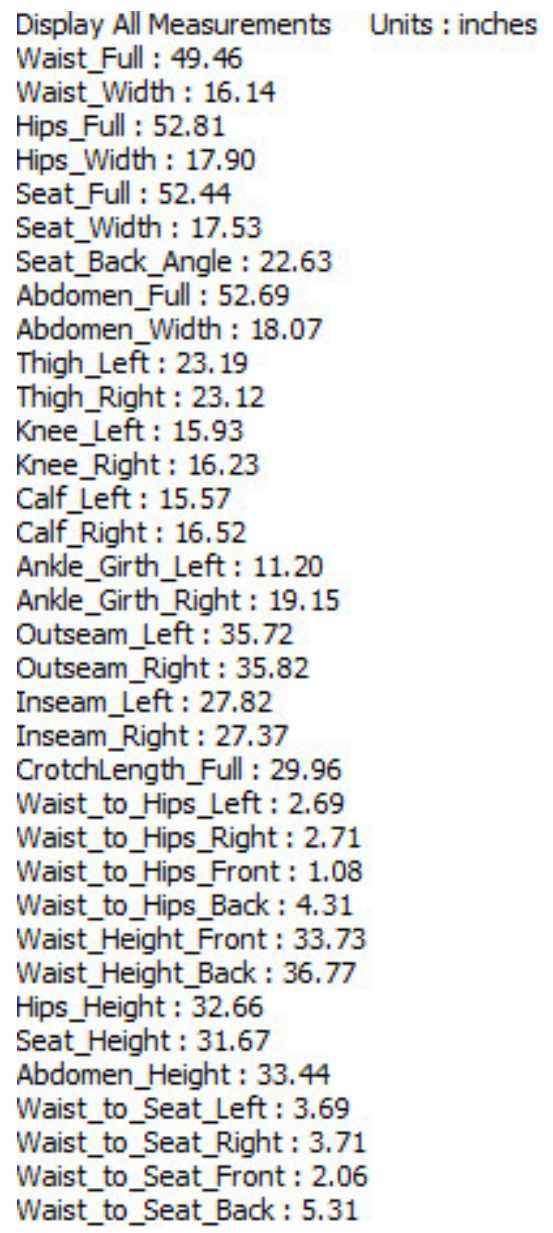

3-Mo Post-Operative Lower Body Measurement

Figure 6. Pre- and Post-Operative Lower Body Measurement Comparisons

As a result of the abdominoplasty procedure, many of the lower body measurements have been reduced. The waist circumference has been reduced over 4 inches. The abdomen circumference has been reduced by nearly 6 inches. The "Crotchlength_Full" is a contoured linear measurement from the small of the back where the scanner software locates the "back of waist" landmark, traversing the "crotch" of the patient and terminates at the "front of waist" landmark. This has been reduced by over 4 inches, as is evidenced by the flatter appearance of the abdominal area.

The patient returned to the clinic for two more follow-up visits. She was scanned at each visit. These 3D body models were appended to her 3D digital archive.

Case 2 presents a situation where the patient presents with her right breast removed. Her left breast is becoming pendulous and ptotic. The right and left arms of the 3D body model have been hidden for greater torso emphasis.

The surgeon is to perform a reconstruction of the left breast and reduce the right breast. Figure 7 presents the results returned from applying the breast measurement template to the pre-operative 3D body model. This template normally assists the surgeon in evaluating breast asymmetries. The key measurements for this are the "Bust_Prominance" and "FrontNeck2Bust" right and left measurements. The "Bust_Prominance" is a contoured curve beginning at the axial fold of the breast and extends across the maximum breast projection and terminates at the inframammary fold. The "FrontNeck2Bust" measurement is a linear, but not contoured measurement that begins at the suprasternal notch and terminates at the maximum bust projection (See Figure 4). 


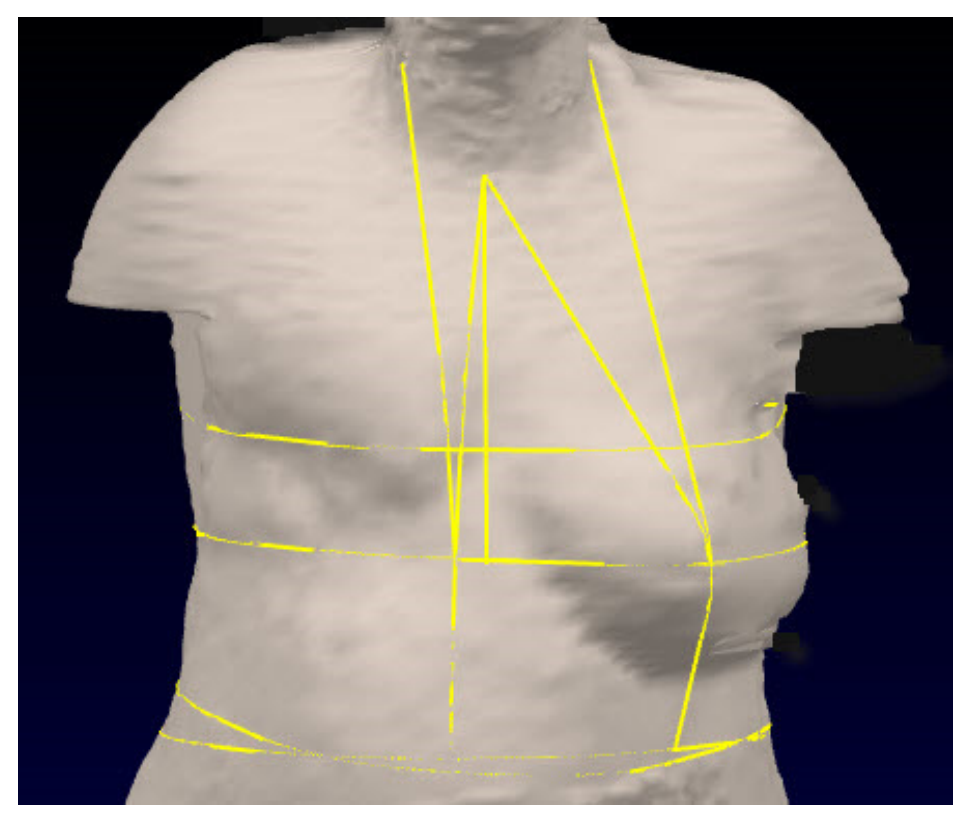

Pre-Operative 3D Body Model in Surface Mode

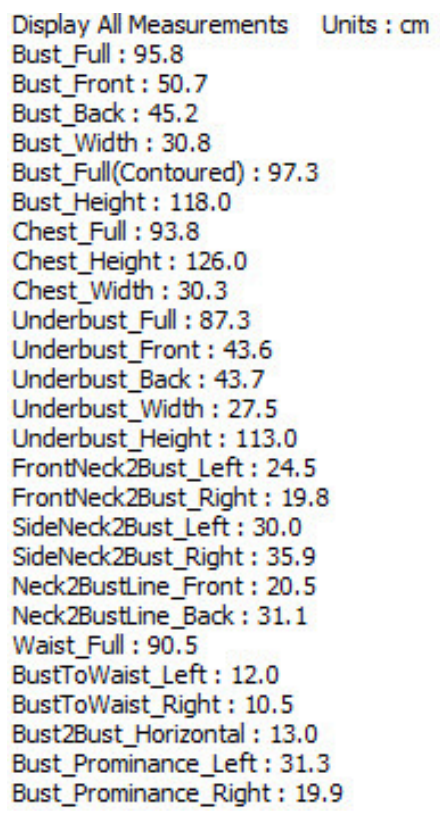

Pre-Operative Bust Measurements

Figure 7. Pre-Operative 3D Body Model and Extracted Bust Measurements, units in cm

In this case, the values returned from the measurements of the right breast area reflect its absence. If the shape of the patient's left breast was desired for replication, the left breast measurements could be used as a guide for reconstruction of the right breast. However, the surgeon elected to reduce the left breast, minimizing the tissue needed from a donor area to reconstruct the right breast.

Figure 8 displays the first phase of reconstructing the right breast. A temporary implant acting as a tissue expander has been inserted. The same measurement template has been applied, and the results are presented to the right of the image. One can observe that the tissue expander in the right breast has made part of the right breast more symmetric with respect to the left breast.

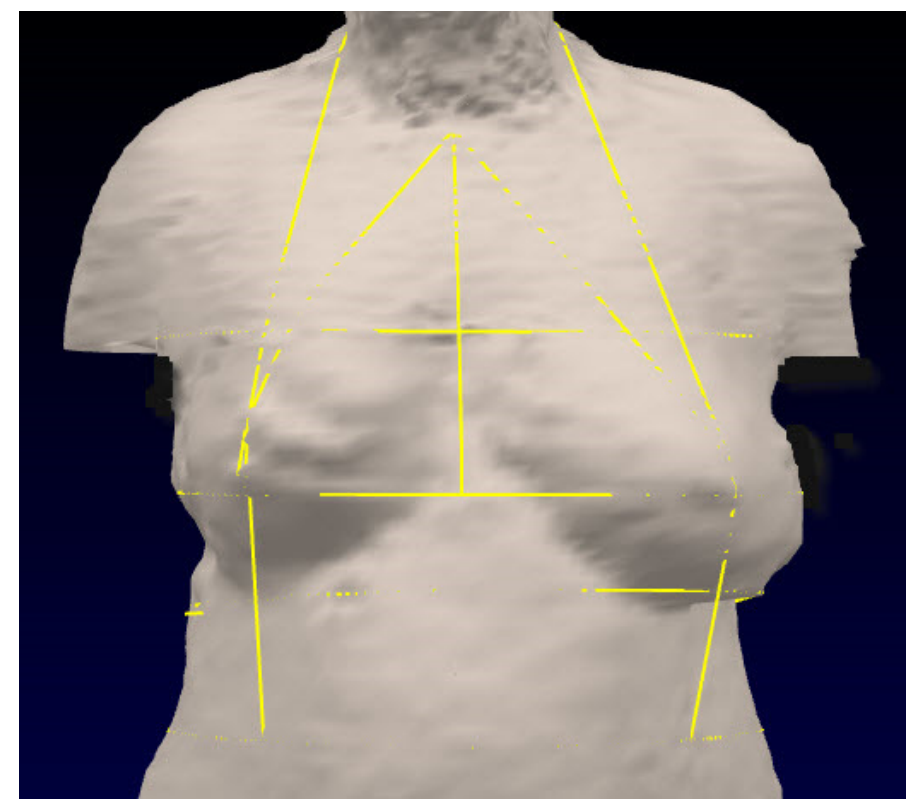

Right Breast with Tissue Expander Implant

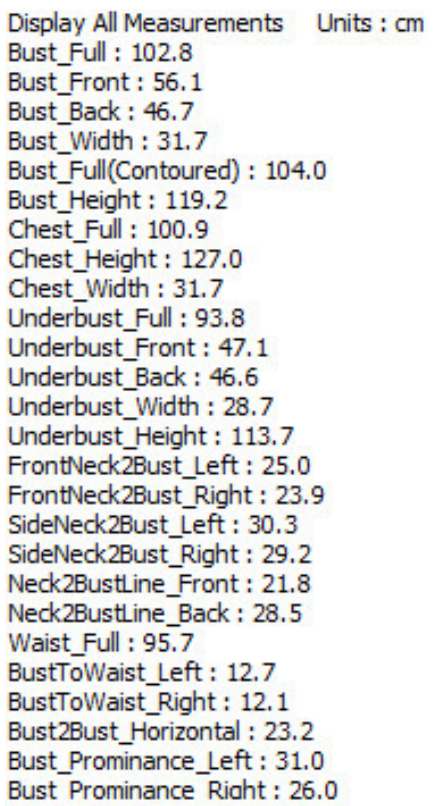

Extracted Measurements

Figure 8. 3D Body Model with Right Breast Tissue Expander and Extracted Measurements

One can begin to observe the right breast beginning to take the desired shape. The "riffles" in the 3D body model image indicate that either the patient was slowly moving during scan acquisition, or that the scanner requires a calibration procedure. 
Figure 9 presents the eventual outcome of the reconstruction of the right breast and the proportional reduction of the left breast. Note the "Bust_Prominance" and "FrontNeck2Bust" left and right measurements are similar in magnitude.

The patient's posture was not erect, as can be seen by the sloping shoulders. Her left arm (not shown) was slightly behind her torso, resulting in a downward twist to the right. This is evident by the right breast appearing lower than the left breast. This might have been corrected by the operator upon further inspection by placing the 3D body model display into "surface mode" (the display defaults to point-cloud mode after the creation and presentation of the 3D body model). However, this is an additional step for the operator, usually a busy nurse, administrator or receptionist. Gross errors can be seen in by viewing the 3D body model in point cloud mode. This scan is certainly usable.

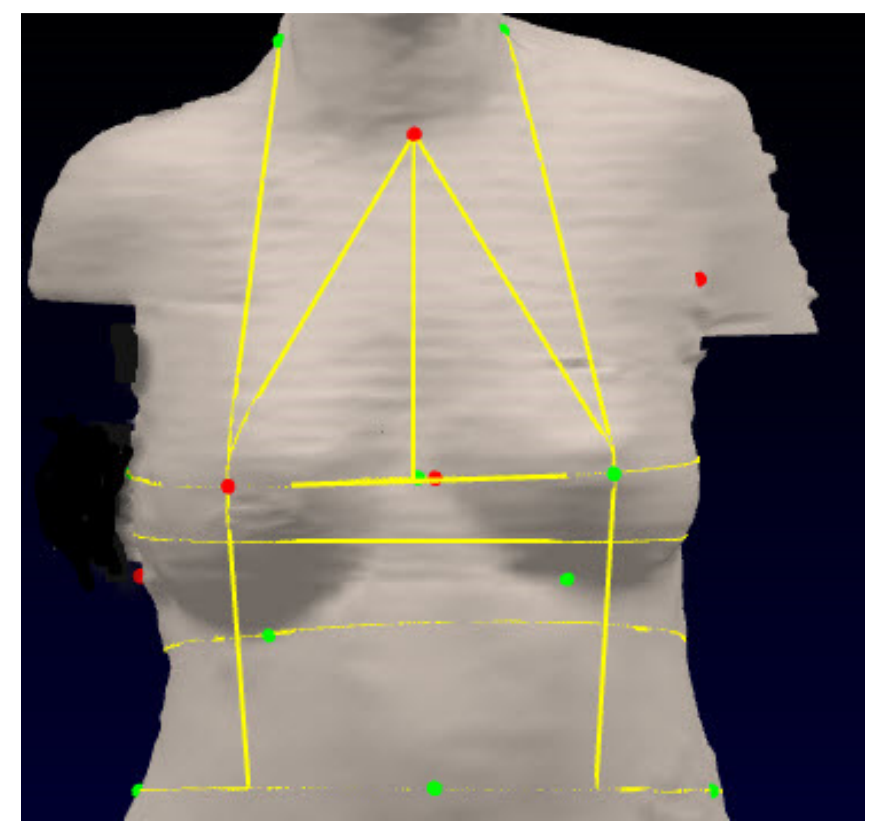

Post-Operative 3D Body Model with Reconstructed Breast

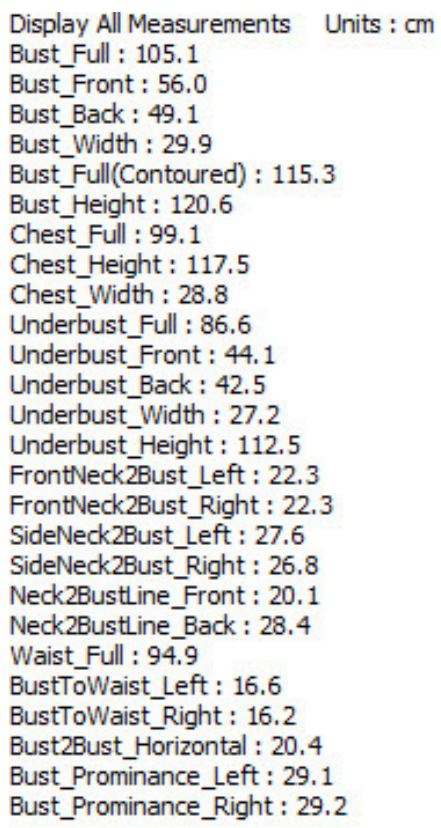

Extracted Measurements

Figure 9. Post-Operative 3D Body Model with Reconstructed Breast and Measurement Extractions

Note the symmetry as indicated by the "Bust_Prominance" and "FrontNeck2Bust" measurement values.

The creation of the pre-operative 3D body model, the 3D body model with the right breast tissue expander implant and the 3D body model of the outcome of the breast reconstruction process form the patient's 3D digital archive. Each 3D body model has value in that it accurately reflects the physical contours of the patient's body at the time of each scan. These are far more useful than traditional 2D photographs, whether printed onto film or stored and presented in a computer image viewing program. The 3D body models can be examined at all angles, and measured with various measurement extraction templates. Measurements extracted from each 3D body model can be tabulated and compared. Together, the viewing of the $3 \mathrm{D}$ body models and the comparisons of the measurements extracted from each 3D body model form a concise history of the physical changes the patient's body underwent resulting from the surgical procedure. It is interesting to note that this sequence of surgeries occurred in 2011. It is 2017 at the time of this writing. It was a simple process of searching the 3D archives to retrieve these 3D body models, present appropriate images and discuss this case.

Case 3 discusses utilizing historical 3D body models for planning additional cosmetic surgery procedures.

It is not uncommon for a patient to desire several cosmetic surgery procedures to be performed over the course of time. An abdominoplasty and adjunct liposuction procedure might be the beginning. A breast lift might follow later, for example. This span of time between surgical procedures may be some years. Although an additional pre-operative 3D scan is acquired prior to the pending surgery, the postoperative 3D body model used to document measurement changes from the initial surgery can be compared with the latest pre-operative 3D body model to evaluate changes to the body contours that may have occurred between surgeries. 
The patient may have gained or lost weight, as would be indicated by a scale reading, but the surgeon would not know which area of the body had been affected by weight gain or loss simply by looking at the difference in weight values.

By applying a general measurement template containing common circumferential measurements to the last post-operative 3D body model and the pre-operative 3D body model acquired for the pending surgery, the surgeon and the patient can understand the physical changes that have taken place in the interim.

This speaks well of the 3D body scanner as a tool for general fitness and health monitoring, outside of its use in the medical arena.

The case presented for the following discussion spans 11 years and several cosmetic surgeries. In each case, a pre-operative 3D body model and at least one post-operative 3D body model were generated by the 3D body scanner. These body models were helpful in evaluating the pre-operative body contours of the patient prior to each surgery, as well as documenting the physical changes resulting from each procedure. Collectively, the series of 3D body models generated over this period also documented physical changes to the body associated with the aging process, which is outside the purview of surgery, yet ever-present.

Figure 10 displays a series of images from the 3D body models of a patient who underwent a pectoral implant procedure followed by a succession of lipoplasty operations.

Each body model image is presented at a 45-degree angle viewed in surface mode. Since these 3D body models are archived, one may apply different measurement profiles to extract measurements of interest. One may also view these 3D body models from different angles. A breast measurement template might be applied to evaluate the results of the pectoral implants, or a lower body measurement profile might be used for evaluating the results from the lipoplasty procedures.

The patient might not be interested in any of these measurement profiles. Rather, he would be more interested in receiving before and after common circumferential measurements displayed in inches.

Since the 3D body model is independent of the measurement profile applied, all can be accommodated.

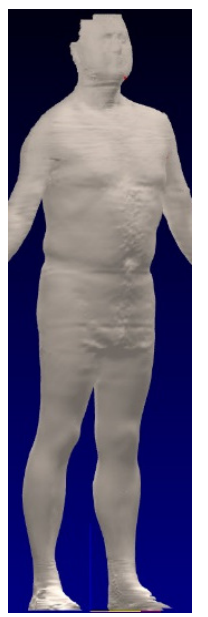

8.25 .05 Preop

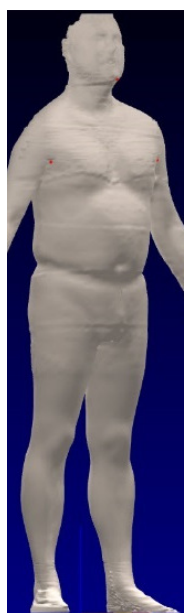

10.06.05 Post Pectoral Implants
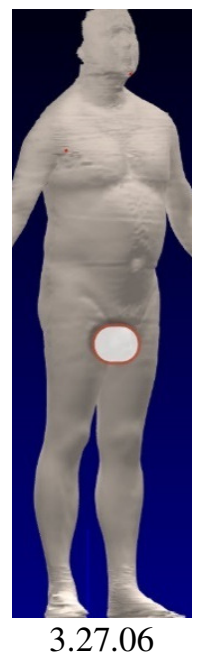

Pre

Lipoplasty No. 1

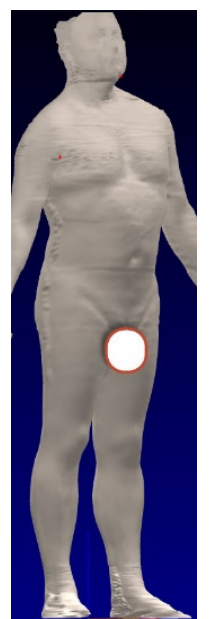

9.18.06

6 -Mo. Post Lipoplasty No. 1

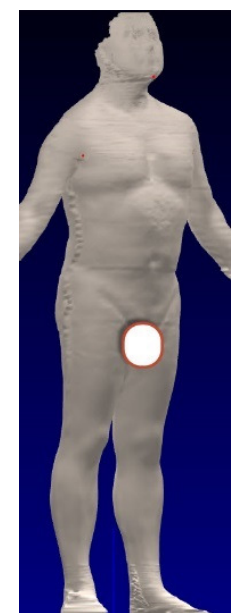

11.27.06 6-Wk Post Lipoplasty No. 2

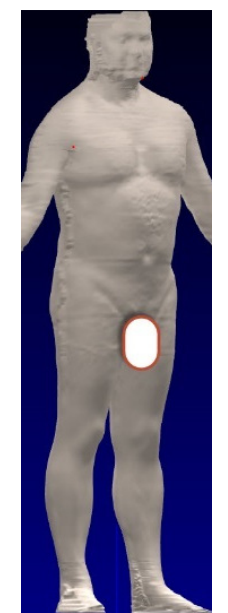

2.05.07

4-Mo. Post

Lipoplasty

No. 2

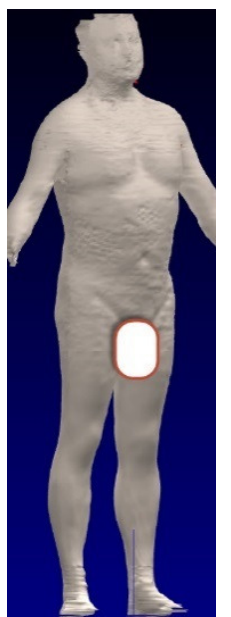

4.30.07

6-Mo. Post Lipoplasty No. 2

Figure 10. 3D Body Models and Date of Acquisition for Specific Cosmetic Surgery Procedures

The patient returned about one year after the scan taken on 4.30 .07 for an additional lipoplasty procedure on his bilateral flanks. In January of 2011 a "mini-tuck" abdominoplasty was performed. These successive 3D body models are depicted in Figure 11. 


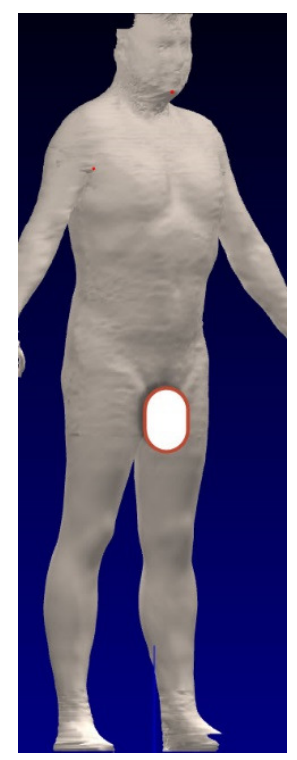

4.14.08

2-Mo. Post

Lipoplasty \#3

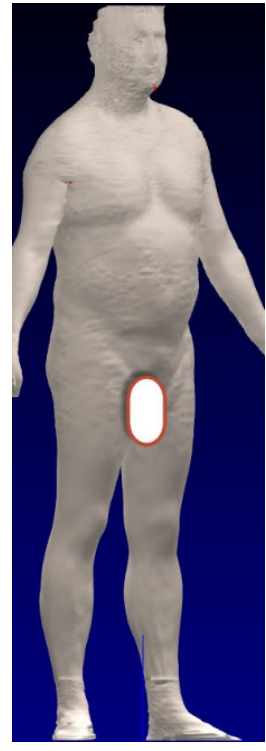

1.17.11

Pre-Bilateral Flank Mini-Tuck

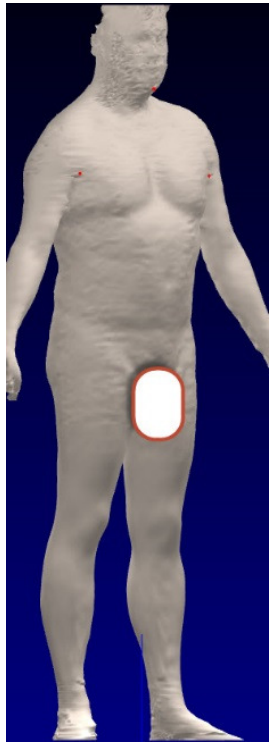

2.21.11

1-Mo. Post Mini-Tuck

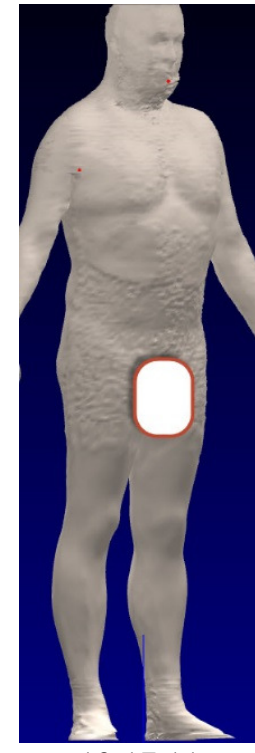

10.17.11

10-Mo. Post Mini-Tuck

Figure 11. A Succession of 3D Body Models Reflecting Surgical Outcomes from 2008 - 2011

The patient returned in August of 2013 for an additional lipoplasty procedure. In September of 2015, the patient underwent gluteal implant surgery. In July of 2016, the patient underwent a procedure to exchange pectoral implants.

The last scan on record was acquired on 8.8.16.

Figure 12 presents the last four successive 3D body models, their dates of acquisition and the surgical procedure associated with each 3D body model.

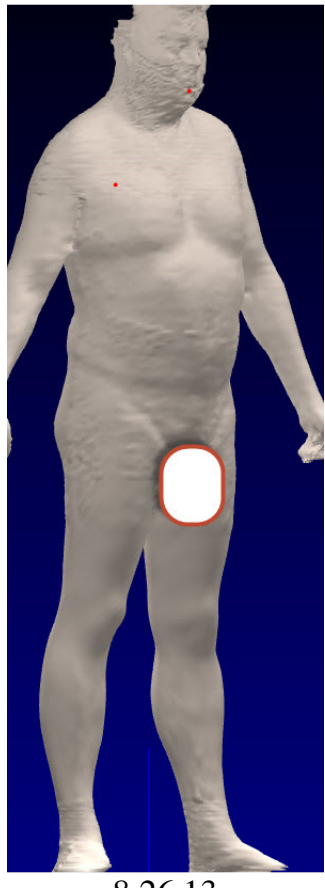

8.26 .13

Pre Lipoplasty No. 4

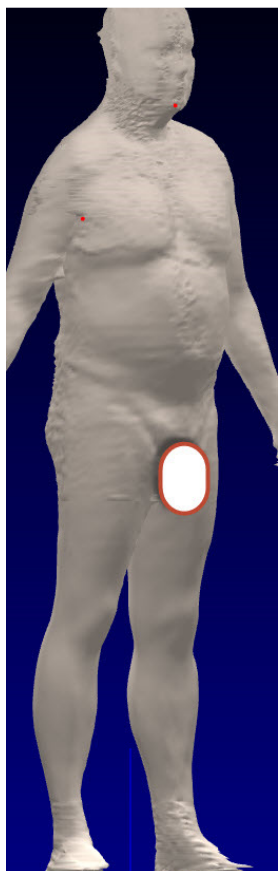

9.3.15

Pre-Gluteal Implants

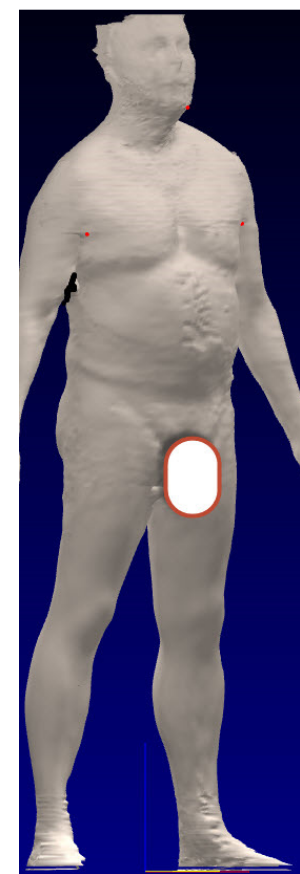

7.11 .16

Pre-Pectoral Implant Exchange

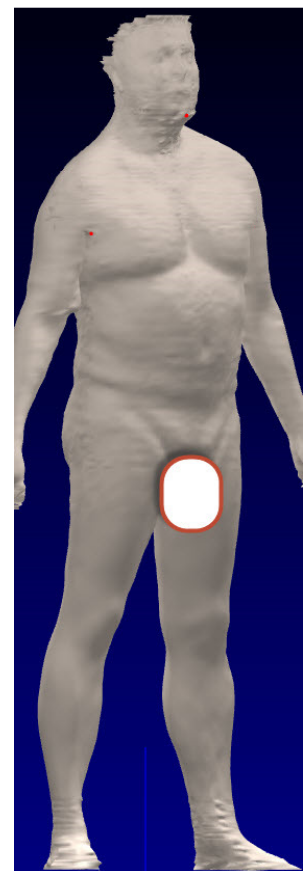

8.8.16

Final Post-op

Figure 12. Succession of 3D Body Models Associated with Surgical Procedures 2013 - 2016 
This collection of fifteen 3D body models acquired from 3D scans over the course of 11 years is quite remarkable. It not only documents the pre- and post-operative contours of the body at the time each scan was captured, it also tells the collective history of the physical shape of the patient. These 3D body models, combined with information found in the traditional medical record, such as weight, clinical blood work and medical prescriptions taken at the time of each procedure combine to give a comprehensive and unique form of medical history.

Figure 13 displays a comparison of common circumferential measurements extracted from the first 3D body model acquired 8.25 .05 , from what visually appears to be the "optimal" physical appearance 3D body model acquired 4.14.08 and the final post-operative 3D body model acquired 8.8.16. For convenience, the measurements extracted are displayed in inches.

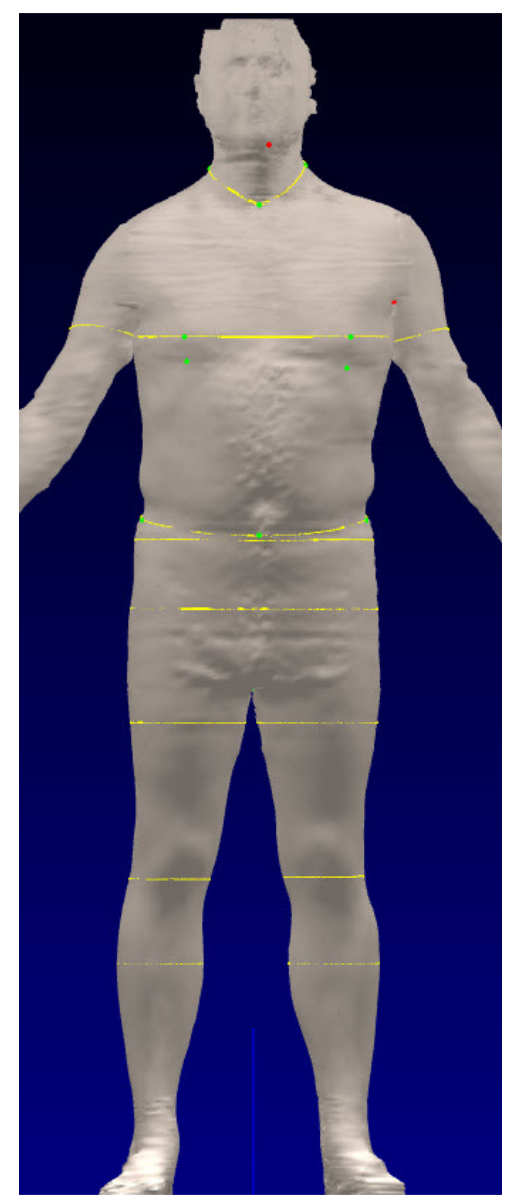

8.25 .05

Display All Measurements Neck_Full : 17.06 Bust_Full : 43.21

Waist_Full : 37.00 Abdomen Full : 38.57

Hips_Full : 39.93

Right_Biceps : 12.62

Left_Biceps : 12.20

Thigh_Left : 21.44

Thigh_Right : 21.40

Knee_Left : 15.30

Knee_Right : 15.23

Calf Left : 15.92

Calf_Right : 15.75

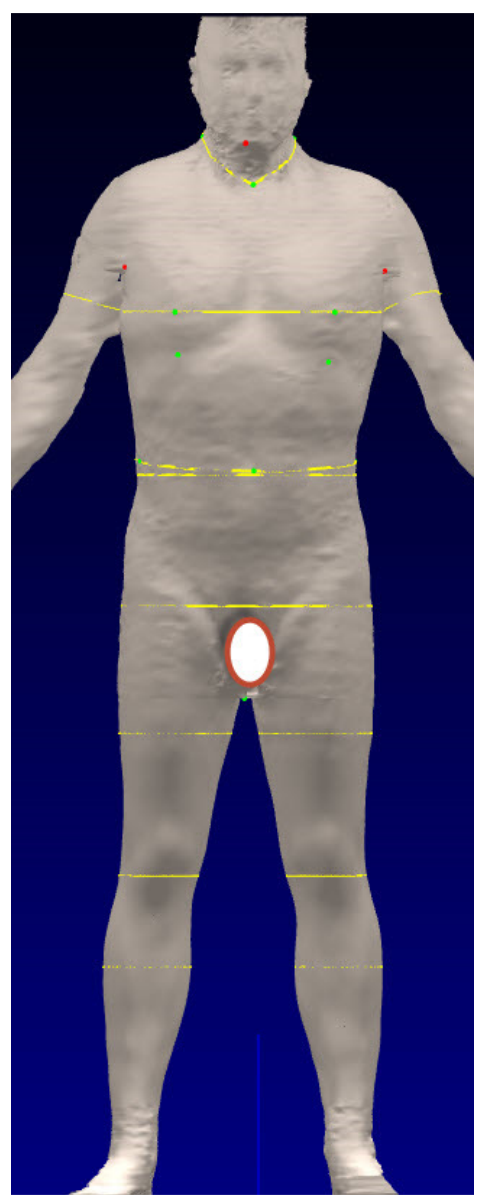

4.14 .08

Units : inche Display All Measurements Neck_Full : 17.20 Bust_Full : 43.46 Waist_Full : 36.14 Abdomen_Full : 36.54 Hips_Full : 39.54 Right_Biceps : 12.09 Left_Biceps : 12.23 Thigh Left : 20.72 Thigh_Right : 20.63 Knee_Left : 15.04 Knee_Right : 14.96 Calf_Left : 15.74

Calf_Right : 15.63

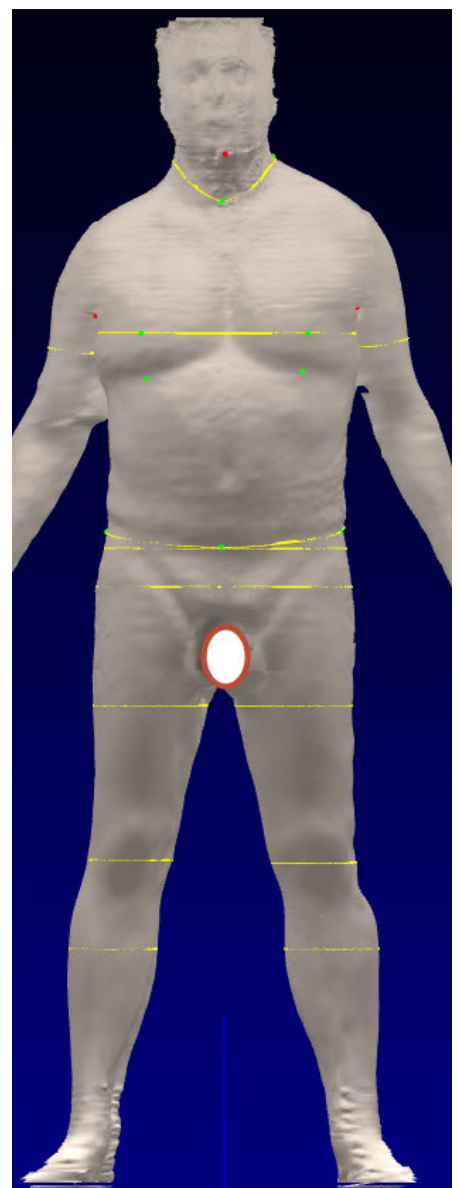

8.8 .16

Units : inche Display All Measurements Units : inche Neck_Full : 17.40

Bust Full : 44.60

Waist_Full : 37.86

Abdomen Full : 39.76

Hips_Full : 41.52

Right Biceps : 13.36

Left_Biceps : 12.94

Thigh_Left : 21.36

Thigh_Right : 21.04

Knee_Left : 15.42

Knee_Right : 15.57

Calf Left: 16.09

Calf_Right : 15.86

Figure 13. Circumferential Measurement Comparisons Between Pre-op, "Visually Optimal" and Final 3D Body Models

Thus, we quickly observe the cumulative visual effects of multiple cosmetic procedures, changes in common circumferential measurements and the aging process over a time span of 11 years. 


\section{Summary and conclusion}

This paper has presented examples of the usefulness of a 3D digital archive created by using a 3D whole-body scanner. Pre-operative 3D body models that are the beginning of a patient's 3D digital archive have been used by the surgeon to evaluate body contours and plan for certain procedures. Post-operative 3D scanning helps to validate the surgical results. All 3D body models created by 3D scanning are archived, and can be retrieved and measured using multiple measurement extraction templates without affecting the integrity of the 3D body model. 3D body models created from our original 3D scanner, that is, from 2002, can be retrieved and measured using current (2017) measurement software provided by 3D scanning system vendors. It is interesting to note that the scan series presented in the last example, which ranged from 2005 until 2016 were acquired by three separate 3D whole-body scanners. As new versions of the 3D scanner became available, the older models were retired. It is evident from the images of $3 \mathrm{D}$ body models presented that continuity has been maintained, along with the faithfulness of the measurement extractions.

3D digital archiving is in its infancy. Today, the 3D body models are separate from the patient's medical records. However, electronic medical records continue to evolve. Flexible formats are being introduced that allow multiple medical devices to export their data directly into these records. There is nothing special about the 3D body model format, since these are readily exported to many common data modeling programs. The 3D body model format could be easily accommodated as the conversion and migration to electronic medical records continues.

We began using a 3D whole-body scanner in a clinical environment in late 2002. The scanner footprint was too large to be housed in an examination room. It was set up in a large storage room. The price of the original scanner was too expensive for it to be consider for general clinical use. Within three years, the footprint of the scanner was reduced to the point where it could be placed in an examination room. The price of the scanner was reduced by half. Two years later, another version of the 3D scanner was available. The footprint of this version was reduced to 5 feet by 4.5 feet, and could comfortably fit into an examination room and still leave enough space for chairs, a coat rack and a desk. The price of this version of the 3D scanner halved again.

In the very near future, "smart mirrors" that hang on the wall will begin to take the place of booth-type 3D scanners. These eventually will be located in an exam room next to the common stadiometer. It is also possible that patients will begin taking 3D scans of themselves since 3D depth sensor chip cameras are becoming standard issue on the latest smart phones.

3D digital archiving, given its utility, is not limited to the surgical examples discussed above. Perhaps its most powerful application will be in the general practice, where 3D scanning can begin at an early age. A patient is usually weighed at each visit. Imagine if a 3D body scan is acquired that same time. The individual's medical history record would be greatly enhanced. This 3D data could be de-identified and combined with 3D scan information from other similar sources to form a vast information archive by which meaningful multidimensional analysis could occur.

Such an analysis, and its results has the potential to discover or validate new 3D dimensional public health indicators based on shape and space and could complement and eventually replace BMI, which relies solely on height and weight.

It is approaching the time that sensor technology for 3D scanning systems will be cost effective and readily available. The next step will be for the industry to agree on sensor-layer protocols, led by an international standards body such as the IEEE. In effect, this will allow sensors to be replaced without retiring the entire scanning system, as had to be done in the past.

This step, once completed, will open the door for an enterprising vendor to seek and secure FDA approval as a Class 2 medical device for a compact, affordable and easy-to-use 3D scanning system without running the risk of technological obsolescence and recertification.

At this point, when this does eventually happen, it is quite possible that the use of a 3D body scanning device will become as commonplace in a clinical environment as a stethoscope. 


\section{References:}

Integrating a 3D Body Scanner into an Active Bariatric Surgery Clinic: Practical Experiences, History, Tips and Pitfalls. David Stefan, M.S.E.E., David A. Gilbert, M.D., Stephen D. Wohlgemuth, M.D. Paper ID 562014 3D Body Scanning Conference. Lugano, CH. October 2014.

This paper details the history of the $3 D$ whole-body scanners employed by The Hague Center for Cosmetic Surgery. It includes a discussion of the many issues that needed to be resolved before $3 D$ scanning of patients became integrated into the workflow of the clinic's personnel. There is a discussion of best practice protocols that are in use for the 3D scanner in a clinical setting.

Breast Augmentation Virtual Surgery Using 3D Body Scanning: Bridging the Gap between Patient Expectations and Surgical Practicalities. David A. Gilbert, M.D. Paper ID 552013 3D Body Scanning Technologies, Long Beach, CA, USA. November 2013.

This paper details the early efforts to develop an effective measurement template for use on breast augmentation and breast reduction patients. It also gives insight into the ability of the scanner to export $3 D$ body models to CAD programs, and how it is possible to perform "virtual surgery" to predict the outcome of breast augmentation and breast reduction procedures prior to conducting the surgery.

Theory and Practical Steps to Introducing a new 3D Public Health Indicator to replace BMI Using Existing Population-based Multidimensional Reference Measurement Sets. David Stefan, M.S.E.E., David A. Gilbert, M.D., Stephen D. Wolgemuth, M.D. Paper ID 55. 2014 3D Body Scanning Conference. Lugano, CH. October 2014.

This paper outlines the development of a "adiposity" indicator that uses surface area and volume, in effect, determining the amount of "space" an individual occupies. It also details an algorithm and scale to mathematically determine the shape of the individual from certain scanner measurements.

Practical Advice: Navigating the Landscape for Using Current 3D Body Scanners in a Medical Environment. Do's, Don'ts and "Maybes." David Stefan, M.S.E.E., David A Gilbert, M.D. Paper ID 23 2016 3D Body Scanning Conference. Lugano, CH. November 2016.

This paper investigates the FDA certification process for a 3D scanner. It includes classifications of medicals devices, reviews the path for interim approval for research on human subjects, and presents the steps required for commercialization once the research has been completed. 